\title{
THERMAL POST-BUCKLING OF SLENDER ELASTIC RODS WITH DIFFERENT BOUNDARY CONDITIONS
}

\begin{abstract}
R. F. Solano ${ }^{\mathrm{a}}$,
and M. A. Vaz $^{\mathrm{b}}$

a PETROBRAS S. A.

Departamento de Engenharia

Av. Almirante Barroso, $81-11^{\circ}$ andar CEP. 20031-004, Rio de Janeiro, RJ, Brasil rsolano@petrobras.com.br

${ }^{\mathrm{b}}$ Universidade Federal do Rio de Janeiro Departamento de Engenharia Naval e Oceânica Av. Brigadeiro Trowpowisk, s/n CEP. 21945-970, Rio de Janeiro, RJ, Brasil murilo@peno.coppe.ufrj.br

ABSTRACT

This paper presents mathematical formulation, critical buckling temperature and analytical and numerical solutions for the thermal post-buckling behavior of slender rods subjected to uniform thermal load. The material is assumed to be linear elastic, homogeneous and isotropic. Furthermore, large displacements are considered hence the formulation is geometrically nonlinear. Three different boundary conditions are assumed: (i) double-hinged non-movable, (ii) hinged non-movable at one end, whereas at the other end longitudinal displacement is constrained by a linear spring, and (iii) doublefixed non-movable. The governing equations are derived from geometrical compatibility, equilibrium of forces and moments, constitutive equations and strain-displacement relation, yielding a set of six first-order non-linear ordinary differential equations with boundary conditions specified at both ends, which constitutes a complex boundary value problem. The buckling and post-buckling solutions are respectively accomplished assuming infinitesimal and finite rotations. The results are presented in nondimensional graphs for a range of temperature gradients and different values of slenderness ratios, and it is shown that this parameter governs the rod post-buckling response. The influence of the boundary conditions is evaluated through graphic results for deformed configuration, maximum deflection, maximum inclination angle and maximum curvature in the rod.
\end{abstract}

Keywords: Elastic Rods, Thermal Buckling, Thermal Post-Buckling.

\section{NOMENCLATURE}

A cross-sectional area

C constants

$E \quad$ Young's modulus

$K_{s}, k_{s}$ spring stiffness

I cross-sectional second moment of inertia

$L, l \quad$ length

$M, m$ bending moment

$n \quad$ sequential integer number

$P \quad$ compressive load

$S, s \quad$ arc-length

$T, t \quad$ temperature

$X, Y$ and $x, y$ Cartesian coordinates

$Y_{\max }, y_{\max }$ maximum vertical displacement

\section{Greek symbols}

$\alpha \quad$ thermal expansion coefficient

$\beta, \theta$ angles

$\Delta T, \Delta t \quad$ temperature variation

$\Delta, \delta$ displacement

$\varepsilon \quad$ strain

$\mathrm{K}, \kappa$ curvature

$\lambda$ rod slenderness ratio

$\pi \quad$ Pi number

\section{INTRODUCTION}

There are many practical cases where buckling and post-buckling of slender rods may occur. The narrow relationship between the thermal buckling of slender components - such as railroad tracks, concrete road pavements, optical fibers, satellite tethers or subsea and buried pipelines - and the buckling of rods has long been recognized. It is therefore of practical design interest to employ analytical formulations. Pipeline instability first works were based on similar problems occurred with raiload tracks (Martinet, 1936 and Kerr, 1974). Analytical and numerical buckling modeling for offshore pipelines has rapidly progressed over the last few years, since the classical design formulations developed by Hobbs (1984) and Hobbs and Liang (1989), which are extensively accepted by industry. Similar studies were presented by Ju and Kyriakides (1998), Chiou and Chi (1996) and Taylor and Gan (1996). The recent demand for higher temperature flowlines and the lack of publications about the subject unleashed the interest to further study this phenomenon. Several papers that describe the structural behavior of pipelines subjected to the action of thermal loading are important to this study.

The problem of elastic stability of rods subjected to mechanical and thermal compressive loads has been well studied since Bernoulli, Euler and Lagrange investigated the classical problem of 
the elastica, i.e., the equilibrium configurations of inextensible rods under axial compression. Love's (1944) seminal textbook on theory of mathematical elasticity has been extensively used in many fields of applied mechanics, establishing the basis for most research on the equilibrium of elastic rods. Some papers were published on buckling and post-buckling behavior obtaining solutions for the differential equation that governs the elastic line of an initially straight slender rod (the elastica problem) subjected to different compressive loads and boundary conditions (Theocaris and Panayotounakos, 1982; Stemple, 1990; Wang, 1997; Filipich and Rosales, 2000 and Vaz and Silva, 2002).

The problem of elastic stability of rods subjected to thermal loads and mechanical compressive loads are substantially different and in fact not as many articles have been published regarding thermal buckling of rods. Buckling and post-buckling behavior in the sense of Koiter were treated within the framework of the general branching theory of discrete systems. Coffin and Bloom (1999) developed an elliptic integral solution for the post-buckling response of a linear-elastic and hygrothermal beam fully restrained against axial expansion. They assumed linear thermal straintemperature relationship and solved the set of differential equations for the undeformed configuration, hence two coupled integral elliptic equations needed to be simultaneously solved. Based on the exact non-linear geometric theory for extensible rods and using a shooting method, a computational analysis for the thermal post-buckling behavior of rods with axially non-movable pinnedpinned ends as well as fixed-fixed ends was proposed by $\mathrm{Li}$ and Cheng (2000). More recently, $\mathrm{Li}$ et al. (2002) presented a mathematical model for the postbuckling of an elastic rod with pinned-fixed ends when a quasi-static increasing temperature is applied. Using the shooting method in conjunction with the concept of analytical continuation, the non-linear boundary value problem consisting of ordinary differential equations was numerically solved. The results showed that the critical buckling temperature and the post-buckled rod configuration were sensitively influenced by the slenderness ratio. Finally, Vaz and Solano (2003 and 2004) and Solano and Vaz (2004) developed a closed-form analytical solution via uncoupled elliptic integrals for the buckling and post-buckling analysis of slender elastic rods subjected to uniform thermal loads.

This paper investigates the buckling and postbuckling response of an initially straight slender rod made of linear elastic material. A temperature gradient is assumed uniform along the rod and expansion is prevented by different boundary conditions. The analytical solution is obtained by uncoupled elliptic integrals, which are derived from the governing equations in the deformed configuration, and numerical technique employs a classical Runge-Kutta high order solution to solve the set of non-linear ordinary differential equations. This study may be qualitatively expanded to pipelines and other slender structures subjected to thermal loads.

\section{MATHEMATICAL FORMULATION}

Consider a uniformly heated slender rod with ends subject to different boundary conditions in its initial and buckled configurations, as shown in Fig. 1. Whereas in Fig. 1a the rod has non-movable doublehinged ends (bi-pinned), in Fig. 1b one edge is considered non-movable and the other edge is limited by a linear spring of stiffness constant $K_{S}$, which restrains the longitudinal expansion. Finally in Fig. 1c the rod has non-movable double-fixed (biclamped) ends.

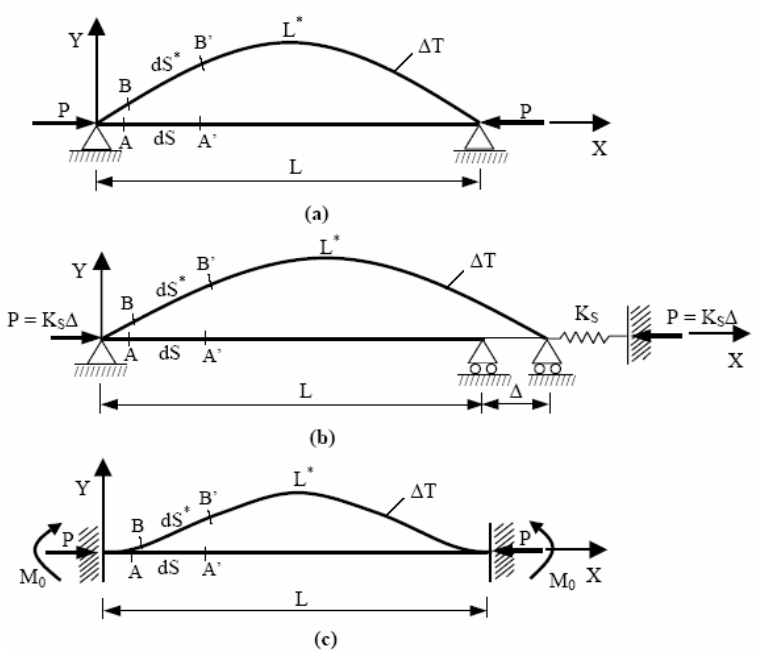

Figure 1. Schematic of a Heated Elastic Rod.

In this paper $(X, Y)$ constitute the rod central line Cartesian coordinates, $\Delta T$ is the uniform temperature gradient, $\mathrm{P}$ is the compressive load arising from the expansion constraint, $M_{0}$ is the end constrained moment, $\mathrm{S}$ is the arc-length, $S^{*}$ is the deformed arclength, $L$ and $L^{*}$ are respectively the initial and deformed rod length, and $\Delta$ is the edge lateral displacement.

The governing equations are derived from the geometrical compatibility, equilibrium of forces and moments, constitutive equations and straindisplacement relation, following the development presented by Vaz and Solano (2003 and 2004) and Solano and Vaz (2004) considering the infinitesimal deformed element of the rod (see Fig. 2).

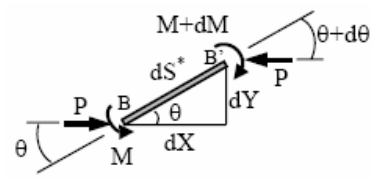

Figure 2. Infinitesimal Element of the Deflected Rod. 
Where $\mathrm{M}$ is the bending moment, $\theta$ is the angle formed by the curve tangent and the longitudinal axis.

Furthermore, non-dimensional variables may be conveniently defined by using the following relations: $L^{*}=l^{*} L \quad S=s L, \quad X=x L, \quad Y=y L$, $S^{*}=s^{*} L, \quad \mathrm{~K}=\kappa / L, \quad \lambda^{2}=L^{2} A / I, \quad P=p E I / L^{2}$, $M_{0}=m E I / L, \quad K_{S}=k_{s} E I / L^{3}, \quad \Delta=\delta L \quad$ and $\Delta T=\Delta t / \lambda^{2} \alpha$. Where: $\mathrm{K}$ is the curvature, $E$ is the Young's modulus, $I$ is the cross-sectional second moment of inertia, $A$ is the cross-sectional area, $\lambda$ is the rod slenderness ratio and $\alpha$ is the thermal expansion coefficient.

Finally, assuming linear elastic, homogeneous and isotropic materials (constitutive relations given by Hooke's Law), and still considering the state of pure bending, the governing equations for slender rods subjected to a uniform temperature gradient are written as:

$$
\begin{gathered}
\frac{d x}{d s^{*}}=\cos \theta \\
\frac{d y}{d s^{*}}=\sin \theta \\
\frac{d \theta}{d s^{*}}=\kappa \\
\frac{d s}{d s^{*}}=\frac{1}{(1+\varepsilon)} \\
\frac{d \kappa}{d s^{*}}=-p \sin \theta \\
\frac{d p}{d s^{*}}=0
\end{gathered}
$$

Where:

$$
\varepsilon=\frac{\Delta t}{\lambda^{2}}-\frac{p}{\lambda^{2}} \cos \theta
$$

is the central line strain, defined as the ratio between the elongation in deformed configuration and its initial length.

In summary, a slender rod tends to expand when subjected to a temperature gradient $\Delta t$ and, consequently, a compressive load $p$ appears if movement of the ends is constrained. Hence, the total strain is given by the addition of the thermal strain and the strain due to the compressive load ( $\varepsilon=\varepsilon_{T}+\varepsilon_{c}$ ). The first term on the right hand side of Eq. (1.7) defines the thermal strain for materials whose strain-temperature dependence is linear.

Associated to the governing equations (1) the following boundary equations should be fulfilled:

$$
x(0)=y(0)=y\left(l^{*}\right)=0
$$

$$
\begin{gathered}
\kappa(0)=x\left(l^{*}\right)-1=\kappa\left(l^{*}\right)=0 \\
\kappa(0)=x\left(l^{*}\right)-1-\delta=\kappa\left(l^{*}\right)=0 \\
\theta(0)=x\left(l^{*}\right)-1=\theta\left(l^{*}\right)=0
\end{gathered}
$$

Where Eqs. (2.2), (2.3) and (2.4) represent respectively bi - pinned, pinned - spring constrained and bi - clamped boundary conditions. Furthermore Eq. (2.1) must be satisfied for the three boundary conditions investigated.

Hence the influence of the slenderness ratio and degree of edge mobility (i.e., spring stiffness) on the critical buckling load and temperature on the rod post-buckled deformed configuration may be analytically and numerically calculated.

\section{CRITICAL BUCKLING TEMPERATURE}

The determination of the critical buckling load is found applying the governing equations for the rod in a slightly deformed configuration. As the rotation $\theta$ is assumed small compared to unity, $\cos \theta \cong 1$ and $\sin \theta \cong \theta$. Consequently, the governing equation may be reduced to:

$$
\frac{d^{4} y}{d x^{4}}+p \frac{d^{2} y}{d x^{2}}=0
$$

A general solution for the homogeneous differential equation (3) with constant coefficients is:

$$
y(x)=C_{1} \sin (\sqrt{p} x)+C_{2} \cos (\sqrt{p} x)+C_{3} x+C_{4}
$$

\section{Double-hinged non-movable (bi-pinned) ends}

The application of boundary conditions yields $C_{2}=C_{3}=C_{4}=C_{1} \sin (\sqrt{p})=0$, and to avoid trivial solution $C_{1}$ must be different from zero, which can be satisfied if $\sin (\sqrt{p})=0$ and $\sqrt{p}=n \pi$, where $n$ is a positive integer. The smallest eigenvalue in this case corresponds to $n=1$, i.e., the first buckling load corresponds to:

$$
p_{c}=\pi^{2}
$$

Subjected to a uniform temperature increase, the rod tends to expand, but until it reaches the critical buckling load, its strain is zero $(\varepsilon=0)$, hence:

$$
\Delta t-p=0
$$

Equation (5) can be substituted in Eq. (6) to find the critical buckling temperature:

$$
\Delta t_{c}=p=\pi^{2}
$$




\section{Longitudinal displacement constrained by a linear spring}

Applying the boundary conditions yields $C_{2}=C_{3}=C_{4}=C_{1} k_{s} \delta \sin \left[(1+\delta) \sqrt{k_{s} \delta}\right]=0$, and to avoid a trivial solution $(1+\delta) \sqrt{k_{s} \delta}=n \pi$. The smallest eigenvalue corresponds to $n=1$, hence the critical lateral displacement for first buckling mode is given by:

$$
\delta_{c}=\frac{(w-2)^{2}}{6 w}
$$

where: $w=\left[8+108 \frac{\pi^{2}}{k_{s}}+\frac{12 \pi}{k_{s}} \sqrt{12 k_{s}+81 \pi^{2}}\right]^{1 / 3}$

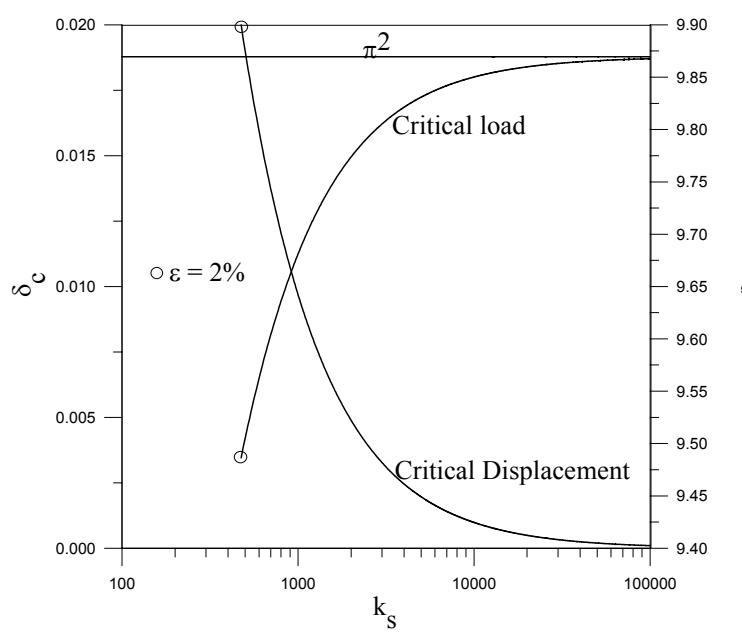

Figure 3. Critical Displacement and Load as a Function of the Spring Stiffness.

In Fig. 3 the critical displacement and buckling load are presented as a function of the spring stiffness. As the spring stiffness increases the end displacement and the buckling load tend to zero and $\pi^{2}$, respectively. On the other hand as $k_{s}$ tends to zero, $\delta_{c}$ grows to infinity and the critical buckling load tends to zero. However, note that the rod strain has been limited to $2 \%$, which gives $k_{s}=474.32$, $\delta_{c}=0.02$ and $p_{c}=9.4864$.

Until the critical buckling load $\left(p_{c}\right)$ is reached a uniformly heated rod tends to expand maintaining its straight configuration (i.e., $\varepsilon=\delta_{c}$ ), hence from Eq. (1.7):

$$
\Delta t=\delta_{c}\left(k_{s}+\lambda^{2}\right)
$$

Note that three parameters control the critical buckling temperature: the spring stiffness $k_{s}$, the rod slenderness ratio $\lambda$ and the critical lateral displacement $\delta_{c}$ being a function of $k_{s}$ through Eq. (8).
The physical and geometrical rod properties should be carefully selected to ensure practical and real meaning to the analysis, and concomitantly avoiding violation of assumptions included in the mathematical formulation. Therefore, high temperatures and strains should not be considered. Furthermore, the parametric study was conducted for rod slenderness ratios $\lambda=50,100,150$ and 200 .

Figure 4 presents the critical buckling temperature as a function of the spring stiffness for four rod slenderness ratios. As the spring stiffness increases the boundary conditions tend to doublehinged non-movable and as expected the critical temperature tends to $\pi^{2}$.

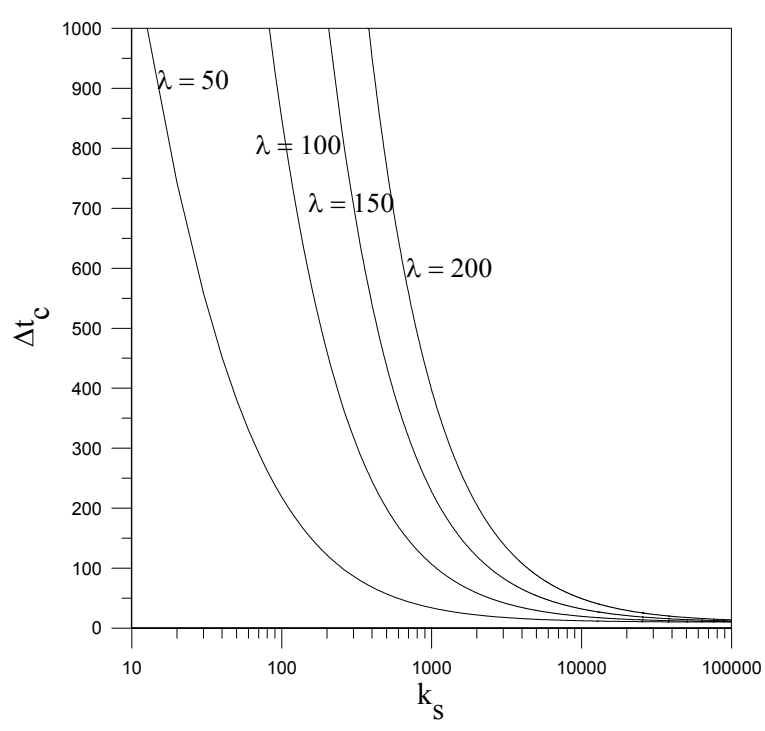

Figure 4. Critical Temperature as a Function of the Spring Stiffness.

\section{Double-fixed non-movable (bi-clamped) ends}

Application of respective boundary conditions yields a homogeneous equation system and for a nontrivial solution it may be shown that either $\sin (\sqrt{p} / 2)=0 \quad$ or $\quad \tan (\sqrt{p} / 2)=\sqrt{p} / 2$. Consequently, the smallest eigenvalue is $\sqrt{p}=2 n \pi$, where $n$ is a positive integer. The smallest eigenvalue corresponds to $n=1$ :

$$
p_{c}=4 \pi^{2}
$$

The strain is zero at the buckling condition, hence:

$$
\Delta t-p=0
$$

Equation (10) can be substituted in Eq. (11) to find the critical buckling temperature:

$$
\Delta t_{c}=p=4 \pi^{2}
$$




\section{ANALYTICAL AND NUMERICAL SOLUTIONS}

\section{Analytical Solution}

For the boundary conditions: (i) double-hinged non-movable and (ii) hinged non-movable and constrained by a linear spring, a closed-form analytical solution for the thermal post-buckling of slender elastic rod uniformly heated is developed via complete elliptic integrals derived from the governing equations in the deformed configuration, following similar works developed by Vaz and Solano (2003 and 2004) and Solano and Vaz (2004). The material is assumed linear elastic and its thermal straintemperature relationship is linear. It is more convenient to work with the slope angle $\theta$ (Bažant and Cedolin (1991)), so the non-dimensional differential Eqs. (1.3) and (1.5) yield:

$$
\frac{d^{2} \theta}{d s^{* 2}}=-p \sin \theta
$$

The solution of the non-linear ordinary differential equation (13) had been earlier solved by Lagrange (a kinetic analogy of columns; Love [8]). Integrating Eq. (13) and applying the boundary conditions at the ends of the rod (i.e., $\theta(0)=-\theta\left(l^{*}\right)=\beta$ and $\left.\kappa(0)=\kappa\left(l^{*}\right)=0\right)$ yield:

$$
\frac{d \theta}{d s^{*}}=-\sqrt{2 p(\cos \theta-\cos \beta)}
$$

Recurring to familiar trigonometric identities to rewrite the Eq. (14), separating and changing variables $(\sin \theta / 2=c \sin \phi$, where $c=\sin \beta / 2)$ and after some algebraic manipulation followed by an integration yields the deformed rod length:

$$
l^{*}=\frac{2}{\sqrt{p}} \int_{0}^{\pi / 2} \frac{d \phi}{\sqrt{1-c^{2} \sin ^{2} \phi}}
$$

The slender rod deflected configuration may be obtained from the non-dimensional Eqs. (1.1) and (1.2) calculating the $x$ and $y$ coordinates respectively:

$$
\begin{gathered}
x=\frac{1}{\sqrt{p}} \int_{\phi_{0}}^{\pi / 2} \frac{1-2 c^{2} \sin ^{2} \phi}{\sqrt{1-c^{2} \sin ^{2} \phi}} d \phi \\
y=\frac{2 c}{\sqrt{p}} \cos \phi_{0}
\end{gathered}
$$

And $-\pi / 2 \leq \phi_{0} \leq \pi / 2$. Since $\kappa=-p y$ the $\operatorname{rod}$ curvature at the deformed configuration may now be readily obtained:

$$
\kappa=-2 c \sqrt{p} \cos \phi_{0}
$$

For the boundary condition double-hinged nonmovable, symmetry implies that the point of maximum displacement occurs for $x\left(l^{*} / 2\right)=1 / 2$, so one may calculate $p$ for this condition as a simple application of Eq. (16.1):

$$
p=\left[2 \int_{0}^{\pi / 2} \frac{1-2 c^{2} \sin ^{2} \phi}{\sqrt{1-c^{2} \sin ^{2} \phi}} d \phi\right]^{2}
$$

Similarly, for one edge limited by a linear spring, symmetry implies that the point of maximum displacement occurs for $x\left(l^{*} / 2\right)=(1+\delta) / 2$, so one may calculate $\delta$ for this condition as a simple application of Eq. (16.1):

$$
\delta=\frac{(W-2)^{2}}{6 W}
$$

where: $W=\left[8+108 \frac{I}{k_{s}}+\frac{12 \sqrt{I}}{k_{s}} \sqrt{12 k_{s}+81 I}\right]^{1 / 3}$ and $I=\left(2 \int_{0}^{\pi / 2} \frac{1-2 c^{2} \sin ^{2} \phi}{\sqrt{1-c^{2} \sin ^{2} \phi}} d \phi\right)^{2}$

Therefore for each deformed configuration (which is related to a temperature gradient), i.e., for a given slope $\beta, c=\sin \beta$ is calculated, and consequently $p\left(p=k_{s} \delta\right.$ for edge limited by a linear spring) from Eqs. (18.1) and (18.2). Finally, it is possible to find the coordinates $(x, y)$ and curvature $\kappa$ along the rod from Eqs. (16.1), (16.2) and (17).

The temperature gradient associated with the deformed configuration may be obtained considering the Eq. (1.7). Thus:

$$
\Delta t=\lambda^{2}\left(l^{*}-1\right)+2 \sqrt{p} \int_{0}^{\pi / 2} \frac{\left(1-2 c^{2} \sin ^{2} \phi\right)^{2}}{\left(1-c^{2} \sin ^{2} \phi\right)^{1 / 2}} d \phi
$$

This expression may be readily evaluated once $p$ and $L^{*}$ are known.

\section{Numerical Solution}

It is difficult to obtain analytical solutions to the rod with bi-clamped ends. Three boundary conditions are given at each rod end, which characterizes a twopoint boundary value problem. Several techniques have been employed for this problem (e.g. finite element methods, finite difference schemes and energy methods). Solutions via the shooting method with direct integration are conveniently employed in linear or non-linear problems when only one parameter is required for interpolation but they become rather complex if two conditions are sought in non-linear systems. 
However, a simple but robust way to transform a boundary into an initial value problem is available in Mathcad (2000) through the following procedure: (a) initial missing values are guessed; (b) the boundary value endpoints are specified; (c) the set of differential equations are defined; (d) a load function which returns the initial conditions is established; (e) a score function to measure the distance between terminal conditions and desired terminal conditions is employed; (f) the equivalent initial conditions are finally calculated. From this point, a classical RungeKutta high order solution may be employed to solve the set of non-linear ordinary differential equations.

\section{POST-BUCKLING RESULTS ANALYSIS}

The closed-form solution is implemented through a computational program developed in the mathematical software Mathcad (2000) and a parametric study is carried out with the purpose of analysing the results. The most significant results regarding the phenomenon of rod thermal postbuckling with (i) bi-pinned and (iii) bi-clamped are presented for typical values of slenderness ratio: compressive load (Fig. 5a), maximum deflection (Fig. 5b), maximum inclination angle (Fig. 5c) and maximum curvature (Fig. 5d).

Once the critical buckling load is reached and the temperature is progressively increased, the compressive force considerably decreases, as it can be observed at Fig. 5. The maximum rod deflection $\left(y_{\max }\right)$, which occurs at $x\left(l^{*} / 2\right)=1 / 2$, increases with temperature as shown at Fig. 5b. The maximum inclination angle $\left(\theta_{\max }\right)$ also increases with temperature (see Fig. 5c) but it occurs at the rod ends. The maximum curvature (Fig. 5d) occurs at the middle of the rod and also increases, in modulus, as temperature is progressively increased.

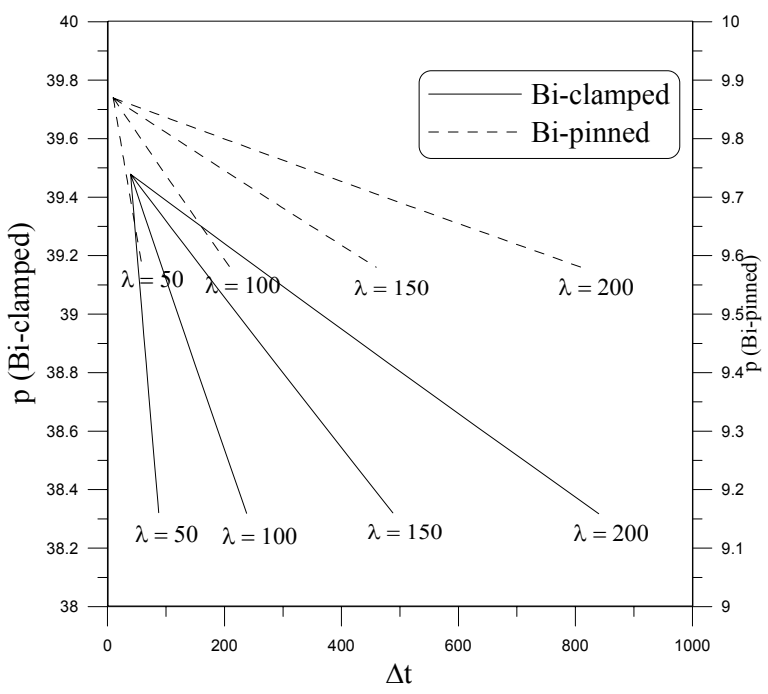

Figure 5a. Compressive Load.

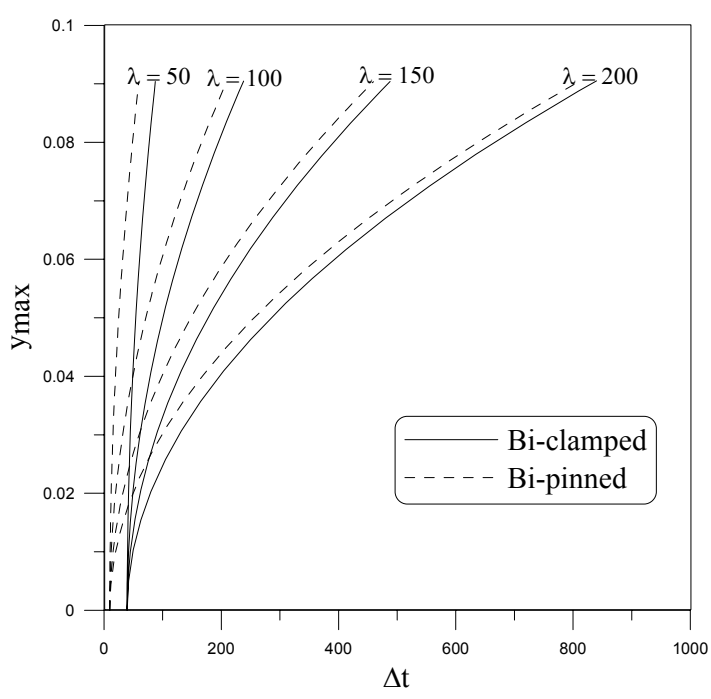

Figure 5b. Maximum Deflection.

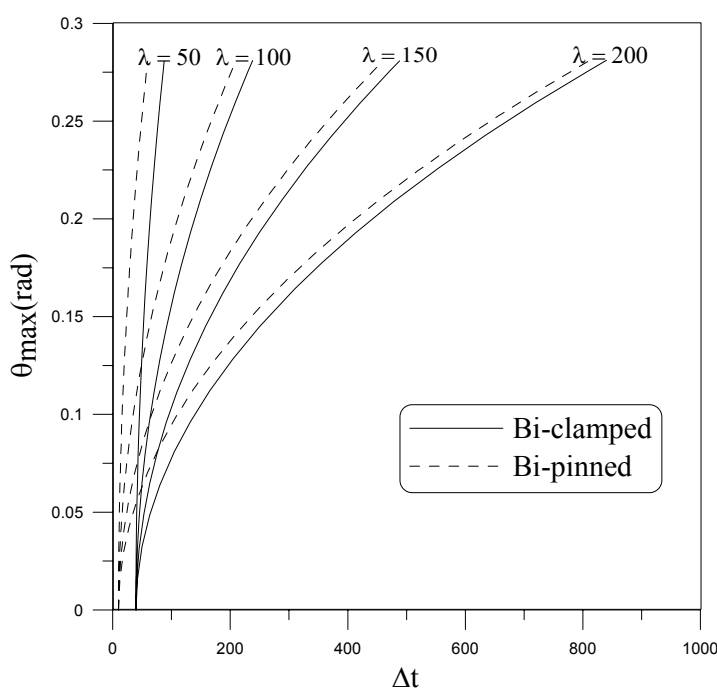

Figure 5c. Maximum Angle.

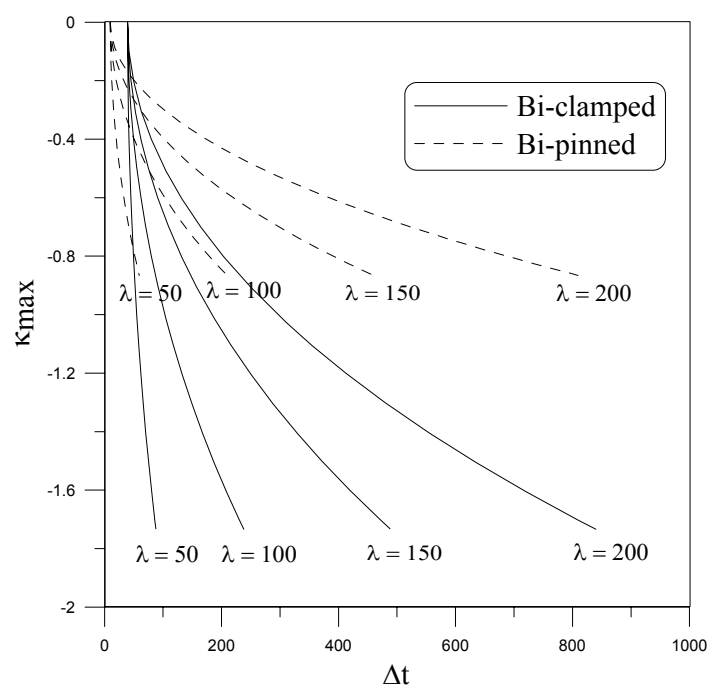

Figure 5d. Maximum Curvature. 
The results for the rod with (ii) pinned-spring constrained ends are shown for typical values of slenderness ratio and stiffness in Figs. $6 \mathrm{a}$ to $6 \mathrm{~d}$. The maximum deflection occurs at $x\left(l^{*} / 2\right)=(1+\delta) / 2$.

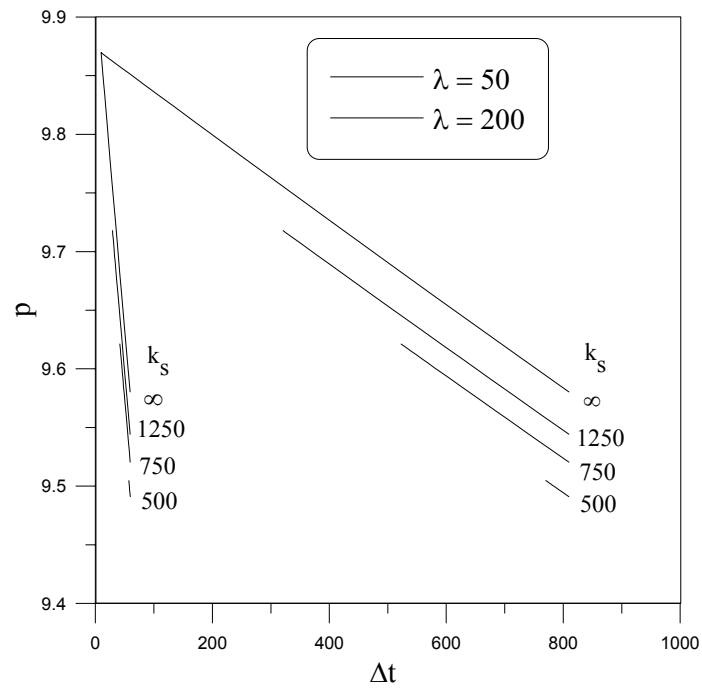

Figure 6a. Compressive Load.

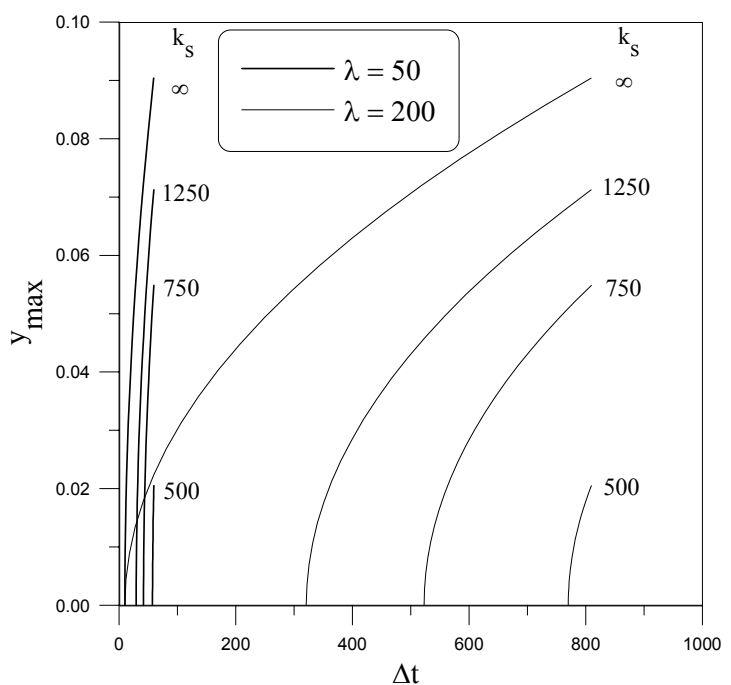

Figure 6b. Maximum Deflection.

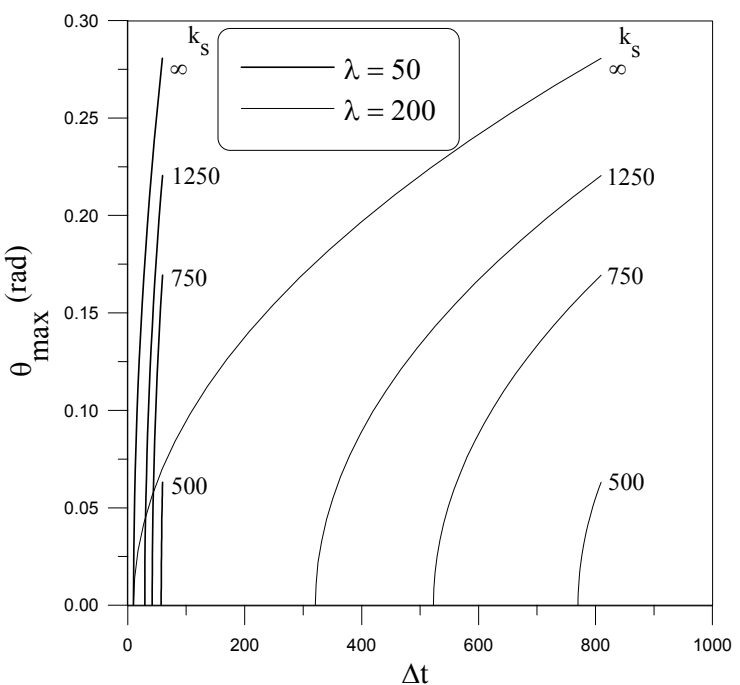

Figure 6c. Maximum Angle.

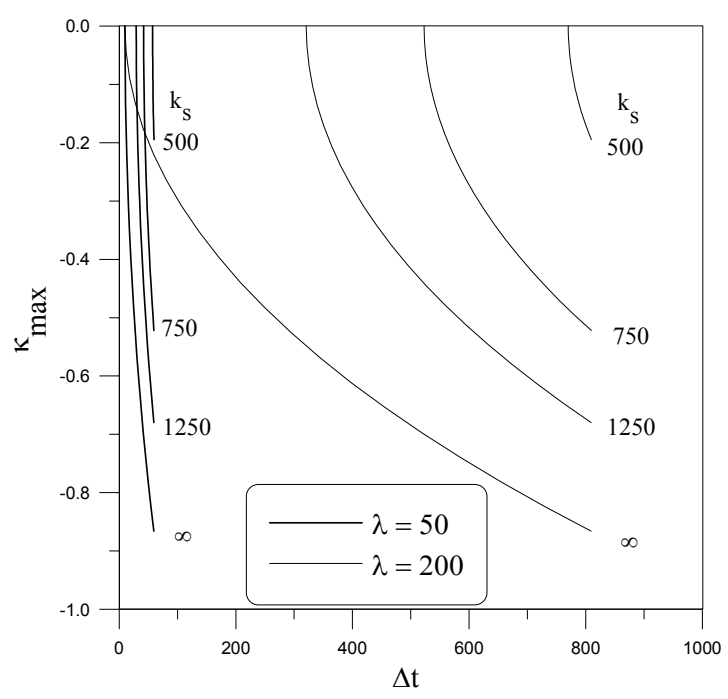

Figure 6d. Maximum Curvature.

Figure 7 presents the rod configurations for a total deformation $\varepsilon=2 \%$ and four spring stiffness $k_{s}=500,750,1250$ and $\infty$. The required temperatures for slenderness ratios $\lambda=50$ and 200 are also displayed.

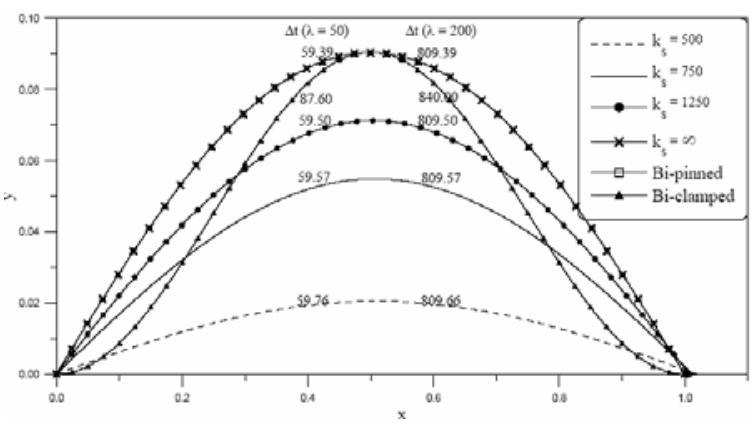

Figure 7. Deflected Configurations for Total Strain $\varepsilon=2 \%$.

\section{CONCLUSIONS}

The mathematical formulations, analytical and numerical solutions presented in this paper have been successfully employed in a two-point boundary value problem governed by a set of six first-order nonlinear ordinary differential equations. The postbuckling analysis of slender elastic rods subjected to uniform temperature variation is highly dependent on the prescribed end conditions.

The governing equations are written in nondimensional form and it is noted that two parameters control the solution: the slenderness ratio and the spring stiffness. The critical buckling displacement, load and temperature are calculated and a parametric study is performed. A closed-form analytical solution via uncoupled elliptic integrals and a numerical technique were developed allowing a complete parametric study. 
The results, presented in non-dimensional graphs, were obtained as a function of the slenderness ratio, temperature and spring stiffness.

\section{ACKNOWLEDGEMENTS}

The authors acknowledge the support from the National Council of Scientific and Technological Development (CNPq), National Petroleum Agency (ANP) and PETROBRAS.

\section{REFERENCES}

Bažant, Z. P. and Cedolin, L., 1991, "Stability of Structures - Elastic, Inelastic, Fracture and Damage Theories", Ed. New York Oxford, pp. 38-45.

Chiou, Y. -J. and Chi, S. -Y., 1996, "A Study on Buckling of Offshore Pipilines", Journal of Offshore Mechanics and Arctic Engineering, vol. 118 , pp. 62-70.

Coffin, D. W. and Bloom, F., 1999, "Elastica Solution for the Hygrothermal Buckling of a Beam", Int. J. Non-Linear Mech., vol. 34, pp. 935-947.

Filipich, C. P. and Rosales, M. B., 2000, "A Further Study on the Post-Buckling of Extensible Elastic Rods", Int. J. Non-Linear Mech., vol. 35, pp. 997-1022.

Hobbs, R. E., 1984, "In-service Buckling of Heated Pipelines", J. Transportation Engineering, vol.110, pp. 175-189.

Hobbs, R. E. and Liang, F., 1989, "Thermal Buckling of Pipelines Closed to Restraints", International Conference on Offshore Mechanics and Arctic Engineering, vol. 5, pp. 121-127.

Ju, G. T. and Kyriakides, S., 1998, “Thermal Buckling of Offshore Pipelines", J. Offshore Mechanics and Arctic Engineering, vol. 110, pp. 355364.

Kerr, A. D., 1974, "On the Stability of the Raiload Track in the Vertical Plane", Rail International, vol. 5, pp. 132-142.

Li, S. R. and Cheng, S. J., 2000, "Analysis of Thermal Post-Buckling of a Heated Elastic Rods", Appl. Math. Mech. (English ed.), vol. 21, pp. 133140.

Li, S., Zhou, Y.- H. and Zheng, X., 2002, "Thermal Post-Buckling of a Heated Elastic Rod with Pinned-Fixed Ends", J. Thermal Stresses, vol. 25, pp. 45-56.

Love, A. E. H., 1944, "A Treatise on the Mathematical Theory of Elasticity", 4th ed., New York: Dover Publications, pp. 399.

Martinet, A., 1936, "Flambement des Voies sans Joints sur Ballast et Rails de Grand Longueur" ("Buckling of Tracks without Joints on Ballast and Very Long Rails" in French), Revue Générale des Chemins de Fer, vol. 55(2), pp. 212-230.

Mathcad, Inc., Mathcad, Mathcad 2001 Professional for PC, MathSoft, Inc.U.S., 2000.
Solano, R. F. and Vaz, M. A., 2004, “Thermal Buckling and Post-Buckling of Slender Rods with Ends Subjected to Different Boundary Conditions", 10th Brazilian Congress of Thermal Sciences and Engineering, Rio de Janeiro.

Stemple, T., 1990, "Extensional BeamColumns: an Exact Theory", Int. J. Non-Linear Mech., vol. 25, pp. 615-623.

Taylor, N. and Gan, A. B., 1996, "Submarine Pipeline Buckling Imperfection Studies", ThinWalled Structures, vol. 4, pp. 295-323.

Theocaris, P. S. and Panayotounakos, D. E., 1982, "Exact Solution on the Non-Linear Differential Equation Concerning the Elastic line of Straight Rod Due to Terminal Loading", Int. J. Non-Linear Mech., vol. 17, pp. 395-402.

Vaz, M. A. and Silva, D. F. C., 2002, "PostBuckling Analysis of Slender Elastic Rods Subjected to Terminal Force", Int. J. Non-Linear Mech., vol. 34, pp. 483-492.

Vaz, M. A. and Solano, R. F., 2003, "PostBuckling Analysis of Slender Elastic Rod Subjected to Uniform Thermal Loads", J. Thermal Stresses, vol. 26, pp. 847-860.

Vaz, M. A. and Solano, R. F., 2004, "Thermal Post-Buckling of Slender Elastic Rod with Hinged Ends Constrained by a Linear Spring", J. Thermal Stresses, vol. 27, pp. 387-380.

Wang, C. Y., 1997, "Post-Buckling of a Clamped-Simply Supported Elastica", Int. J. NonLinear Mech., vol. 32, pp. 1115-1122.

Received: May 18, 2006

Revised: June 18, 2006

Accepted: July 18, 2006 